\title{
Anxiety disorders before birth and self-perceived distress during pregnancy: Associations with maternal depression and obstetric, neonatal and early childhood outcomes
}

\author{
Julia Martini ${ }^{\text {a, }}{ }^{*}$, , Susanne Knappe ${ }^{\text {a,1 }}$, Katja Beesdo-Baum ${ }^{\text {a }}$, Roselind Lieb ${ }^{\text {b,c }}$, Hans-Ulrich \\ Wittchen ${ }^{\mathrm{a}, \mathrm{c}}$ \\ a Institute of Clinical Psychology and Psychotherapy, Technische Universitaet, Dresden, Germany \\ b Institute of Psychology, Epidemiology and Health Psychology, University of Basel, Switzerland \\ c Max Planck Institute of Psychiatry, Munich, Germany
}

\begin{abstract}
Background: Maternal perinatal mental health has been shown to be associated with adverse consequences for the mother and the child. However, studies considering the effect of DSM-IV anxiety disorders beyond maternal self-perceived distress during pregnancy and its timing are lacking. Aims: To examine the role of maternal anxiety disorders with an onset before birth and self-perceived distress during pregnancy for unfavourable maternal, obstetric, neonatal and childhood outcomes. Study design: DSM-IV mental disorders and self-perceived distress of 992 mothers as well as obstetric, neonatal and childhood outcomes of their offspring were assessed in a cohort sampled from the community using the Munich-Composite International Diagnostic Interview. Logistic regression analyses revealed associations (odds ratios) between maternal anxiety disorders and self-perceived distress during pregnancy with maternal depression after birth and a range of obstetric, neonatal and childhood psychopathological outcomes.

Results: Lifetime maternal anxiety disorders were related to offspring anxiety disorders, but not to offspring externalizing disorders. Analyses focussing on maternal DSM-IV anxiety disorders before birth yielded associations with incident depression after birth. In addition, self-perceived distress during pregnancy was associated with maternal depression after birth, preterm delivery, caesarean section, separation anxiety disorder, ADHD, and conduct disorder in offspring.

Conclusion: Findings confirm the transmission of anxiety disorders from mother to offspring. Apart from maternal anxiety, self-perceived distress during pregnancy also emerged as a putative risk factor for adverse outcomes. The finding that maternal anxiety disorders before birth yielded less consistent associations, suggests that self-perceived distress during pregnancy might be seen as a putative moderator/mediator in the familial transmission of anxiety.
\end{abstract}

Keywords: Anxiety disorders, Distress, Pregnancy, Obstetric, Neonate

\section{Introduction}

Research from the past two decades suggests that maternal anxiety, depression, and prenatal psychological distress are associated with maternal mental disorders after birth, and with adverse obstetric, neonatal, and early childhood psychopathological outcomes [1-8]. However, studies often yielded inconsistent results, due to blending of different conceptualizations of anxiety, depression, and self-perceived distress (e.g., considering broad symptom levels vs. DSM-IV disorders) and ignoring the timing of these conditions (e.g., onset of maternal disorders before or after delivery) [4,9-11]. Studies also vary with regard to outcomes of interest in both mothers and their offspring. Hence, after careful inspection of the literature, we aim to disentangle the association of DSM-IV anxiety disorders before birth and 
self-perceived distress during pregnancy with (I) maternal mental disorders after birth, (II) obstetric and neonatal outcomes, and (III) early childhood psychopathology.

\subsection{Maternal DSM-IV anxiety disorders and psychological distress during pregnancy and maternal mental disorders after birth}

The onset of most DSM-IV anxiety disorders is early in life, typically in childhood or adolescence [12,13]. During fertile age, DSM-IV anxiety disorders arewidespread (12-month prevalence inwomen aged 18-34: 17\%, CI: 14\%-20\%) [14]. Though pregnancy is usually considered as a time of relative mental well-being, the dramatic hormonal alterations during this period may change the vulnerability to mental disorders [15].

Prenatal anxiety was found to be an independent risk factor for postpartum depression $[16,17]$. In addition, mostly retrospective case reports show an attenuation of prenatal anxiety symptoms during pregnancy and an increased risk for onset or exacerbation of DSM-IV panic disorder, obsessive-compulsive disorder, and posttraumatic stress disorder after delivery [18]. In contrast, a recent populationbased study indicated no significant alteration of prevalence rates during perinatal period for a range of DSM-IV anxiety disorders [17].

Various attempts have been made to predict postpartum disorders by using questions about putative risk factors and unfavourable conditions during pregnancy [19], such as poor social support, unwanted pregnancy, and marital conflict/problematic partnership [4,20-22]. Most of them describe adversities contributing to perceived distress in the mother. In our study though none of these assessments were used for logistical reasons. Instead, we only asked for perceived distress during pregnancy as a proxy to indicate increased likelihood for maternal mental disorders after birth [23-25].

\subsection{Maternal DSM-IV anxiety disorders and psychological distress during pregnancy and obstetric and neonatal outcomes}

Anxiety symptoms during pregnancy and psychological distress have been reported to be associated with preterm delivery (PTD), low birth weight (LBW), obstetric complications, and pain medication under labour $[1,4,26]$. Notably, PTD and LBW were found to be risk factors for later physical morbidity (diabetes mellitus, heart disease, and hypertonia) [4,27]. However, only few studies systematically evaluated the potential role of manifest maternal DSM-IV anxiety disorders for obstetric and neonatal outcomes apart from perceived psychological distress during pregnancy [1,2]. Posttraumatic stress disorder prior to conception resulted in significantly more pregnancy complications (e.g., preterm contractions and hyperemesis). Antenatal panic disorder was associated with PTD and LBW of the neonate [28-30].

Maternal psychopathology may also increase the risk for intrapartum complications, but studies so far revealed conflicting results $[1,2,4]$.

\subsection{Maternal DSM-IV anxiety disorders and psychological distress during pregnancy and early childhood disorders}

A range of studies indicated associations between elevated levels of maternal anxiety and psychological distress with early behavioural and emotional problems in offspring. Even after controlling for approved confounders (e.g., nicotine consumption and postpartum depression) externalizing problems such as attention deficit/hyperactivity disorder (ADHD), conduct 
disorder (CD), and oppositional defiant disorder (ODD) [3,31-33], but also

behavioural/emotional problems [34,35] and childhood anxiety [36] were more frequently diagnosed in offspring, when mothers were anxious or stressed during pregnancy. Unfortunately, the association with separation anxiety disorder (SAD) has not been investigated, yet. These offspring outcomes have also been shown to increase the risk for other offspring childhood and adolescent disorders such as anxiety, mood, somatoform, and substance use disorders [37-41].

In sum, studies so far suggest that maternal anxiety disorders and psychological distress during pregnancy negatively affect maternal as well as offspring outcomes. However, valid conclusions from these studies are limited due to differences in the definition and operationalization of outcome variables (e.g. confounding of LBW and PTD: many preterm infants show also LBW) and inadequate control of covariates (e.g. comorbid depression) [26]. In addition, assessment of DSM-IV disorders is often hampered by the overlap of somatic pregnancy-related conditions, perinatal distress, and DSM-IV anxiety disorders [1]. The spectrum of mood states during the perinatal period ranges from prenatal distress to depressive and/or anxiety disorders [9-11]. To distinguish between these concepts is of great importance given the possible differences in mother and offspring outcomes, and associated implications for targeted prevention and early intervention [9,11].

The aim of this study was therefore to examine the impact of maternal DSM-IV anxiety disorders before birth and self-perceived distress during pregnancy on I) maternal mental disorders after birth, II) obstetric and neonatal outcomes (mode of delivery, PTD, LBW and APGAR-index) and III) early childhood disorders in offspring (any anxiety disorder, SAD, enuresis, encopresis, ADHD, CD and ODD).

\section{Methods}

The current analyses focus on $\mathrm{N}=992$ mother-child pairs from a representative community sample of the prospective-longitudinal Early Developmental Stages of Psychopathology (EDSP) study. Offspring refer to the younger EDSP-cohort aged 14-17 years at baseline who were followed-up approximately 2 (T1), 4 (T2) and 10 (T3) years after baseline. Details of the design, methods, and assessment of the EDSP study have been reported previously [42,43]. All participants (in cases of aged 18 or younger their parents) provided written informed consent; the EDSP project and its family genetic supplement have been approved by the Ethics Committee of the Medical Faculty of the Technische Universitaet Dresden (No: EK13811).

DSM-IV mental disorders in offspring and mothers were assessed face-to-face with the computer-assisted version of the standardized Munich-Composite International Diagnostic Interview (DIA-X/M-CIDI) [44-46]. For offspring, the lifetime version was used at baseline, the interval version at follow-up. Offspring anxiety diagnoses were cumulated across all available assessment waves (T0, T1, T2 and T3). Mothers were directly interviewed at T1, using the same instrument.

\subsection{Predictors}

When mothers reported onset of a DSM-IV anxiety disorder (including specific phobia, social phobia, generalized anxiety disorder, panic disorder, agoraphobia, obsessive-compulsive disorder and posttraumatic stress disorder) before birth of the index child (subsequently referred to as offspring), they were classified to have 'any anxiety disorder' (ANX). Mothers 
were considered as a case, when onset of ANX was before or during pregnancy. Mean age of onset for ANX was 12.9 years ( $S D=9.4$ years), mean age of recency was 44.1 years $(S D=6.0$ years). All mothers were affected during pregnancy. Mothers without DSM-IV anxiety disorder before birth were classified to have 'no anxiety disorder' (no ANX). Mean age of mothers at delivery was 29.5 years ( $S D=5.2$ years), with no differences between mothers with $(M=29.3$ years, $S D=4.8$ years $)$ and without $(M=29.6$ years, $S D=5.2$ years $)$ an anxiety disorder before birth.

Self-perceived distress during pregnancy (SPD) in mothers was assessed by the question "During pregnancy, how much did you feel strained?” Response was recorded using a four point Likert scale. Answers were dichotomized into 'low SPD' (answer was 'not at all' or 'only little'), and 'high SPD' (answer was 'mild' or 'severe') reflecting substantial distress during pregnancy. In the current sample, SPD was negatively associated with living with a partner (OR=0.8; 95\%CI: $0.7-0.9, \mathrm{p}=0.002)$, and positively associated with problems with the family during pregnancy (OR=1.4; 95\%CI: $1.2-1.6, \mathrm{p}=0.000)$ and unwanted pregnancy (OR=1.4; 95\%CI: 1.0-1.8, $\mathrm{p}=0.031)$.

In addition, the relationship of age of onset of ANX with SPD during pregnancy was examined, since mothers with an onset of ANX during pregnancy may report higher levels of SPD during pregnancy than mothers with an earlier onset of ANX. Relationships were examined using the Area under the Receiver Operating Characteristic (AUC-curve). When age of onset of ANX is unrelated to SPD during pregnancy, AUC is 0.5. An AUC of 1 represents a perfect association (age of onset of ANX perfectly predicts SPD during pregnancy). AUC was 0.48 (AUC onset, SPD) indicating that reported levels of SPD during pregnancy are independent from age of onset of ANX.

To particularly examine the role of maternal ANX and SPD, and to disentangle interpretation of these results from depression, women with (comorbid) DSM-IV depressive disorders before birth were excluded ( $\mathrm{N}=57 / 992)$, resulting in $\mathrm{N}=935$ mothers available for analyses. This approach also permits to investigate the impact of maternal ANX and SPD on incident depressive disorders after birth.

Among those $\mathrm{N}=935$ mothers, $\mathrm{N}=208$ (22.3\%) reported an onset of ANX before birth. $\mathrm{N}=727 / 935(77.8 \%)$ never had a DSM-IV anxiety disorder $(\mathrm{N}=653)$ or reported an onset after birth ( $\mathrm{N}=74)$, and were therefore classified to have 'no ANX' before birth. High SPD during pregnancy was reported by $\mathrm{N}=102 / 935$ (10.9\%).

We first investigated the associations of ANX before birth and SPD during pregnancy with the considered maternal, obstetric, neonatal, and childhood outcomes. Associations between ANX and respective outcomes were controlled for SPD and vice versa, in order to examine the independent contributions of these conditions.

Because these conditions may also occur in combination, we created four mutually exclusive groups to investigate the common effects of maternal ANX before birth and SPD during pregnancy: The four groups were generated through cross-tabulation of maternal diagnostic status and SPD (group 1/reference group: no ANX and low SPD, N=656; group 2: no ANX and high SPD, N=71; group 3: ANX and low SPD, N=177; and group 4: ANX and high SPD, $\mathrm{N}=31$ ).

\subsection{Outcomes}


I) When onset of maternal DSM-IV major depressive episode or dysthymia was after birth, the respective case was classified as depressive disorder after birth. Mean age of onset was 40.6 years ( $\mathrm{SD}=7.2$ years), mean age of recency was 42.9 years ( $\mathrm{SD}=8.9$ years). Mothers were also asked about II) mode of delivery (vaginal delivery, assisted vaginal delivery that is foceps delivery or vacuum extraction, and unplanned caesarean section) and adverse neonatal outcomes (PTD: gestational age less than 37 completed gestation weeks [47], birth weight and APGAR-scoreN7 (APGAR-score is determined by evaluating the newborn on five criteria: Appearance, Pulse, Grimace, Activity and Respiration; APGAR-score ranges from zero to ten)) [48]; and III) early adversities, namely if their child ever had enuresis (at least once per month, for a duration of at least 3 months, at age 5 or older), encopresis (at least once per month, for a duration of at least 3 months, at age 4 or older), CD, ODD, and ADHD (according to DSM-IV criteria). Offspring anxiety disorders (including social phobia, specific phobia, panic disorder, agoraphobia, generalized anxiety disorder, obsessive-compulsive disorder and posttraumatic stress disorder) were directly assessed in offspring using the DIA$\mathrm{X} / \mathrm{M}$-CIDI. DSM-IV SAD was directly assessed in offspring interviews at first follow-up.

\subsection{Statistical analyses}

Results (\%, coefficients) are weighted by age, gender, and geographic location at baseline to match the distribution of the original sampling frame [42]; frequencies (Ns) are reported unweighted. The Stata Software package 10.0 [49] was used to compute robust variances, confidence intervals, and p-values (by applying the Huber-White sandwich matrix) which is required when analyses are based on weighted data [50]. (Multinomial) logistic regressions provided odds ratios (ORs) for associations between maternal diagnostic status and SPD during pregnancy with (I) maternal mental disorders after birth, (II) obstetric and neonatal outcomes, and (III) early childhood disorders. As an exploratory study, no adjustment for multiple testing was applied, because the individual tests were related to individual hypotheses and adjustment would treat them as reflecting a global hypothesis-which is questionable in substantive terms [51].

\section{Results}

Consistent with previous studies, maternal lifetime anxiety disorders were found to be associated with offspring anxiety disorders (including social phobia, specific phobia, panic disorder, agoraphobia, generalized anxiety disorder, obsessive-compulsive disorder and posttraumatic stress disorder) $(\mathrm{OR}=1.4,95 \% \mathrm{CI}$ : 1.1-1.9), but not with mode of delivery, neonatal (PTD, LBW and APGAR), or other childhood outcomes (enuresis/encopresis, CD, ODD, ADHD and SAD). High SPD during pregnancy was unrelated to offspring anxiety disorders, but associated with PTD (OR=3.4, 95\%CI: 1.5-7.9), marginally lower AGPAR $(\mathrm{OR}=2.8,95 \% \mathrm{CI}$ : 0.9-7.9, $\mathrm{p}=0.054)$, and a higher risk for caesarian section $(\mathrm{OR}=1.9,1.1-$ 3.1) as well as offspring $\mathrm{CD}(\mathrm{OR}=5.0,95 \% \mathrm{CI}$ : 1.2-21.3), and ADHD (OR=4.7, 95\%CI: 2.210.0).

Using a strictly prospective approach, the following analyses focus on mothers with an onset of maternal anxiety disorder before birth of their offspring and SPD during pregnancy as a predictor variable (controlled for each other), and maternal, obstetric, neonatal and childhood disorders as outcomes (Table 1). ANX before birth was associated with maternal depressive disorder after birth. SPD was similarly associated with maternal depressive disorder after birth, but also with a higher risk for caesarean section, PTD and early childhood disorders (SAD, ADHD and CD). The associations of SPD with early childhood disorders did not change substantially when additionally controlling for maternal depressive disorder after 
birth, except that the association between SPD and CD was attenuated to nonsignificance (OR=5.6, 95\%CI: 0.9-32.9, p=0.055). Thus, maternal depressive disorder after birth is unlikely to mediate the association between SPD and early childhood disorders.

The examination of the outcomes in the four mutually exclusive groups revealed similar results, with the strongest associations occurring when both ANX and SPD were present. Compared to mothers without ANX and low SPD (reference group, group 1), mothers with high SPD but no ANX (group 2) had a higher risk for caesarean section (OR=2.6, 95\%CI: 1.3-5.0, $\mathrm{p}=0.005$ ). Mothers with ANX and low SPD during pregnancy (group 3) had a higher risk for depressive disorders after birth (OR=2.1, 95\%CI: 1.4-3.2, $\mathrm{p}=0.001$ ) compared to mothers without ANX and SPD (group 1). When both ANX and high SPD (group 4) were reported, a higher risk for maternal depressive disorder after birth (OR=4.8, 95\%CI: 2.1-11.2, $\mathrm{p}=0.000)$, PTD (OR=7.4, 95\%CI: 1.9-29.2, $\mathrm{p}=0.005)$, enuresis and encopresis $(\mathrm{OR}=4.8$, 95\%CI: 1.6-14.2, $\mathrm{p}=0.005)$, ADHD (OR=5.3, 95\%CI: 1.8-16.2, $\mathrm{p}=0.003$ ), and ODD (OR=3.8, 95\%CI: 1.3-11.2, $\mathrm{p}=0.016$ ) was found compared to mothers from the reference group.

Associations between ANX and high SPD (group 4) with psychopathological outcomes in offspring appeared to be almost unaffected by the presence of maternal depressive disorder after birth. That is, all associations except for enuresis and encopresis (OR=3.5, 95\%CI: 0.912.8, $\mathrm{p}=0.062)$ and $\mathrm{ODD}(\mathrm{OR}=1.9,95 \% \mathrm{CI}$ : 0.5-6.8, $\mathrm{p}=0.320)$ remained significant.

\section{Discussion}

Using a population-based cohort sample, we examined the role of maternal ANX before birth and SPD during pregnancy (beyond maternal depressive disorders before birth) for unfavourable maternal, obstetric, neonatal and childhood outcomes.

In line with previous studies [7,12,34,35,37], maternal lifetime anxiety disorders were found to be associated with offspring anxiety disorders, but not with offspring externalizing disorders.

When analyses were restricted to the presence of ANX before birth (controlled for SPD), ANX was unrelated to the investigated outcomes, except for maternal depressive disorder after birth. In addition, high SPD (controlled for ANX) was associated with a particular risk for maternal depressive disorder after birth, PTD, caesarean section, SAD, ADHD, and CD in the offspring. The coincidence of ANX before birth and SPD during pregnancy (group 4) substantially increased the risk for maternal depressive disorder after birth, PTD and psychopathological outcomes in the offspring.

The mother-offspring-transmission of anxiety may be particularly pronounced in the presence of risk factors after birth such as maternal mental disorders. We therefore additionally controlled associations between maternal ANX and SPD and early childhood psychopathological outcomes for maternal depressive disorders after birth. Results remained stable to a large extent indicating that maternal depressive disorder after birth unlikely mediates associations between maternal ANX before birth and SPD during pregnancy with early childhood psychopathological outcomes. Nevertheless, it should be noted that bonding disorders, adverse mother-infant-interaction, or adverse parental rearing behaviour-which were not considered in the current study-may also affect the relationship between maternal ANX, SPD and neonatal and childhood outcomes of their offspring [4,52,53]. 
The strong association of higher levels of SPD with adverse offspring outcomes might be particularly explained by psycho- physiological pregnancy processes. For example, the alteration of the hypothalamic-pituitary-adrenocortical system during pregnancy is involved in the timing of delivery and fetal maturation. Increased sympathetic activation in mothers leading to elevated norepinephrine levels and increased uterine artery resistance might impede the blood flow to the fetus, resulting in LBW and the induction of PTD (for overview see [5456]). Hence, asking for perceived stress during pregnancy such as by "During pregnancy, how much did you feel strained?” holds promise to identify pregnant women at risk for later depressive disorders, obstetric adversities as well as neonatal and early childhood problems in their offspring. Further validation of the predictive power and clinical utility of such a question is however warranted.

The findings of our study should be interpreted with regard to some limitations. Mothers with depressive disorders before birth were excluded from analyses in order to examine the impact of ANX before birth and SPD during pregnancy on disorders after birth apart from concurrent depression, and to investigate the single vs. combined effects of ANX and SPD on incident depressive disorders. We did not exclude mothers with other comorbid disorders to allow for a more representative sample of mothers, and to prevent from limitations in statistical power. Our study did not allow to examine more heterogenous pregnancy processes intensively, such as associations of maternal and offspring psychopathology with gestation problems $[4,36,57]$. Mothers' age at childbirth and age of onset of maternal anxiety disorders were only assessed in measures of years. To minimize errors in determination of age of onset we calculated a proxy for the presence of anxiety disorders before birth and depressive disorders after birth, respectively, by using the age of the mother at interview and her offspring's exact date of birth. However, a small proportion of mothers may have been misclassified as having for instance the anxiety onset before birth when first onset was in fact shortly (within weeks or few months) after childbirth. Due to the low number of directly assessed fathers ( $N=27)$, data on paternal psychopathology were not included. A potentially significant limitation of our study is that distress during pregnancy (SPD) was not assessed by a psychometrically established instrument. Assessment of SPD was based on one item in mothers' interview to serve as a proxy for general evaluation of SPD during pregnancy. Pilot data suggest that this question is associated with psychosocial load. Internal and predictive validity of this approach however have yet to be established.

With these limitations in mind, our findings suggest that the time period prior and during pregnancy is a sensitive developmental niche for both the mother and her offspring in which the foundation for their individual further emotional, behavioural and mental development is laid. Thus, a prospective-longitudinal approach in a high risk sample of women, starting prior to or early in pregnancy is needed to allow for more precise predictions of parental and offspring outcomes. 
Table 1

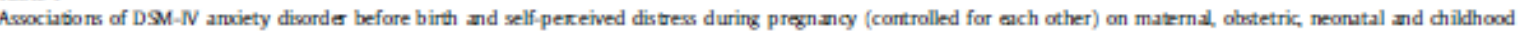
outames.

\begin{tabular}{|c|c|c|c|c|c|c|}
\hline & \multicolumn{3}{|c|}{ ANX $(N=208$, contralled for SPD $)$} & \multicolumn{3}{|c|}{ High $\$ D(N=102$, controlled for $A N X)$} \\
\hline & N & Sw & OR (95acl) & N & Tw & OR (95\%) \\
\hline \multicolumn{7}{|l|}{ M.ternal disorders after birth } \\
\hline Depressian & 56 & 35.7 & $22(1.5-32)$ & 28 & 18.4 & $20(12-3.4)$ \\
\hline \multicolumn{7}{|l|}{ Mode of delivery } \\
\hline Vaginal delivery vs ssisted dedivery & 25 & 11.8 & $1.3(08-22)$ & 10 & 109 & $1.4(06-29)$ \\
\hline Vaginal delivery vs caesarean section & 24 & 120 & a.7 $(04-1.2)$ & 23 & 245 & $23(13-40)$ \\
\hline \multicolumn{7}{|l|}{ Neonxal outromes } \\
\hline APCAR $<7$ & 2 & 43 & $0.4(0.1-1.5)$ & 6 & 16.4 & $23(08-7.0)$ \\
\hline \multicolumn{7}{|l|}{ Offspring outromes } \\
\hline Enuresis/encopresis & 13 & 7.0 & $20(10-41)$ & 4 & 49 & $1.1(0.4-30)$ \\
\hline ADHD & 11 & 5.8 & $19(09-42)$ & 11 & 109 & $45(20-102)$ \\
\hline ODD & 10 & 47 & $1.0(0.5-22)$ & 7 & 75 & $1.9(08-47)$ \\
\hline $\mathrm{CD}$ & 2 & as & a.7 $(0.1-3.8)$ & 3 & 32 & $6.2(1.3-29.3)$ \\
\hline Any mxiety disorder & 84 & 41.4 & $1.3(0.9-1.8)$ & 42 & 41.9 & $1.4(0.9-22)$ \\
\hline
\end{tabular}

Noz: ne not estimated; based on their sye of onset reports, mothers were divided int ANX before vs. fiter bi ith, so goups are mutually exdusive and ORs c.mnot be cakulazed.

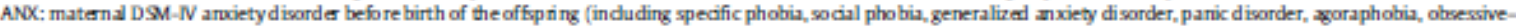
compukive disord es and postraumabic stress disorde L SPD: self-perceived distress during pregrancy.

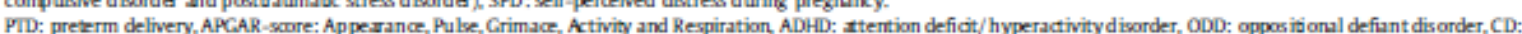

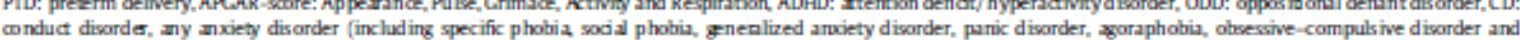
postraumatic stress disorder), SAD: separtion amviety disarder.

$N$ umweigh zed numbers, Iw weighted percentages

OR odds ratio from logistic regression, Q confidence interval

Bold type indictes significance $x p<005$.

\section{Acknowledgements}

This work is part of the Early Developmental Stages of Psychopathology (EDSP) Study and is funded by the German Federal Ministry of Education and Research (BMBF) project nos. 01EB9405/6, 01EB 9901/ 6, EB01016200, 01EB0140, and 01EB0440. Part of the field work and analyses were also additionally supported by grants of the Deutsche Forschungsgemeinschaft (DFG) LA1148/1-1, WI2246/1-1, WI 709/7-1, and WI 709/8-1. Principal investigators are Dr. Hans-Ulrich Wittchen and Dr. Roselind Lieb.

Core staff members of the EDSP group are: Dr. Kirsten von Sydow, Dr. Gabriele Lachner, Dr. Axel Perkonigg, Dr. Peter Schuster, Dr. Michael Höfler, Dipl.-Psych. Holger Sonntag, Dr. Tanja Brückl, Dipl.- Psych. Elzbieta Garczynski, Dr. Barbara Isensee, Dipl.-Psych. Agnes Nocon, Dr. Chris Nelson, Dipl.-Inf. Hildegard Pfister, Dr. Victoria Reed, Dipl.-Soz. Barbara Spiegel, Dr. Andrea Schreier, Dr. Ursula Wunderlich, Dr. Petra Zimmermann, Dr. Katja Beesdo-Baum, Dr. Antje Bittner, Dr. Silke Behrendt and Dipl.-Psych. Susanne Knappe. Scientific advisors are Dr. Jules Angst (Zurich), Dr. Jürgen Margraf (Basel), Dr. Günther Esser (Potsdam), Dr. Kathleen Merikangas (NIMH, Bethesda), Dr. Ron Kessler (Harvard, Boston) and Dr. Jim van Os (Maastricht).

\section{References}

[1] Alder J, Fink N, Bitzer J, Hösli I, Holzgreve W. Depression and anxiety during pregnancy: a risk factor for obstetric, fetal and neonatal outcome? A critical review of the literature. J Matern Fetal 2007;20(3):189-209.

[2] Andersson L, Sundström-Poromaa I, Wulff M, Aström M, Bixo M. Implications of antenatal depression and anxiety for obstetric outcome. Am J Obstet Gynecol 2004;104(3):467-76.

[3] Glover V, O'Connor TG. Antenatal programming of child behavior and neurodevelopment: links with maternal stress and anxiety. In: Hodgson DM, Coe CL, editors. Perinatal programming: early life determinants of adult health and disease. London: Taylor \& Francis; 2006. p. 253-65. 
[4] Halbreich U. The association between pregnancy processes, preterm delivery, low birth weight, and postpartum depressions - the need for interdisciplinary integration. Am J Obstet Gynecol 2005;193(4):1312-22.

[5] Lieb R, Isensee B, Höfler M, Wittchen H-U. Parental depression and depression in offspring: evidence for familial characteristics and subtypes? J Psychiatr Res 2002;36:237-46.

[6] Monk C. Stress and mood disorders during pregnancy: implications for child development. Psychiatr Q 2001;72(4):347-57.

[7] Schreier A, Wittchen H-U, Höfler M, Lieb R. Anxiety disorders in mothers and their children: prospective longitudinal community study. Br J Psychiatry 2008;192:308-9.

[8] Van den Bergh BRH, Mulder EJH, Mennes M, Glover V. Antenatal maternal anxiety and stress and the neurobehavioural development of the fetus and child: links and possible mechanisms. A review. Neurosci Biobehav Rev 2005;29:237-58.

[9] Austin M-P. Antenatal screening and early intervention for "perinatal" distress, depression and anxiety: where to from here? Arch Womens Ment Health 2004;7:1-6.

[10] Austin M-P, Hadzi-Pavlovic D, Leader L, Saint K, Parker G. Maternal trait anxiety, depression and life event stress in pregnancy: relationships with infant temperament. Early Hum Dev 2005;81(2):183-90.

[11] Riecher-Rössler A, Steiner M. Perinatal stress, mood and anxiety disorders: from bench to bedside. Basel: Karger; 2005.

[12] Beesdo K, Knappe S, Pine DS. Anxiety and anxiety disorders in children and adolescents: developmental issues and implications for DSM-V. Psychiatr Clin N Am 2009;32:483-524.

[13] Wittchen H-U, Lieb R, Schuster P, Oldehinkel AJ. When is onset? Investigations into early developmental stages of anxiety and depressive disorders. In: Rapoport JL, editor. Childhood onset of "adult” psychopathology. Clinical and research advances. Washington: American Psychiatric Press; 1999. p. 259-302.

[14] Wittchen H-U, Jacobi F. Size and burden of mental disorders in Europe-a critical review and appraisal of 27 studies. Eur Neuropsychopharmacol 2005;15(4):357-76.

[15] Glover V, Kammerer M. The biology and pathophysiology of peripartum psychiatric disorders. Prim Psychiatry 2004;11(3):37-41.

[16] Heron J, O'Connor TG, Evans J, Golding J, Glover V. The course of anxiety and depression through pregnancy and the postpartum in a community sample. J Affect Disord 2004;80:65-73.

[17] Vesga-Lopez O, Blanco C, Keyes K, Olfson M, Grant BF, Hasin DS. Psychiatric disorders in pregnant and postpartum women in the United States. Arch Gen Psychiatry 2008;65(7):805-15. [18] Ross LE, McLean LM. Anxiety disorders during pregnancy and the postpartum period: a systematic review. J Clin Psychiatry 2006;67(8):1285-98.

[19] Austin M-P, Lumley J. Antenatal screening for postnatal depression: a systematic review. Acta Psychiatr Scand 2003;107:10-7.

[20] Cooper PJ, Murray L, Hooper R, West A. The development and validation of a predictive index for postpartum depression. Psychol Med 1996;26(3):627-34.

[21] O'Hara MW, Swain AM. Rates and risk of postpartumdepression—a meta-analysis. Int Rev Psychiatry 1996;8(1):37-54.

[22] Reid AJ, Biringer A, Carroll JD, Midmer D, Wilson LM, Chalmers B, et al. Using the ALPHA form in practice to assess antenatal psychosocial health. Can Med Assoc J 1998;159(6):677-84.

[23] Cooper RL, Goldenberg RL, Das A, Elder N, Swain M, Norman G, et al. The preterm prediction study: maternal stress is associated with spontaneous preterm birth at less than thirty-five weeks' gestation. Am J Obstet Gynecol 1996;175(5):1286-92.

[24] O'Hara MW, Gorman LL. Can postpartum depression be predicted? Prim Psychiatry 2004;11(3):42-7.

[25] Steiner M. Postnatal depression: a few simple questions. Fam Pract 2002;19(5): 469-70.

[26] Paarlberg KM, Vingerhoest JJM, Passchier J, Dekker GA, Van Geijn HP. Psychosocial factors and pregnancy outcome: a review with emphasis on methodological issues. J Psychosom Res 1995;39(5):563-95.

[27] Erickson K, Thorsen P, Chrousos G, Grigoriadis DE, Khongsaly O, McGregor J, et al. Preterm birth: associated neuroendocrine, medical, and behavioral risk factors. J Clin Endocrinol Metab 2001;86(6):2544-52. 
[28] Banhidy F, Acs N, Puho E, Czeizel AE. Association between maternal panic disorders and pregnancy complications and delivery outcomes. Eur J Obstet Gynecol Reprod Biol 2006;124:47-52. [29] Seng JS, Oakley DJ, Sampselle CM, Killion C, Graham-Bermann S, Liberzon I. Posttraumatic stress disorder and pregnancy complications. Obstet Gynecol 2001;97(1):17-22.

[30] Warren SL, Racu C, Gregg V, Simmens SJ. Maternal panic disorder: infant prematurity and low birth weight. J Anxiety Disord 2006;20(3):342-52.

[31] Burke JD, Loeber R, Birmaher B. Oppositional defiant disorder and conduct disorder: a review of the past 10 years, part II. J Am Acad Child Adolesc Psychiatry 2002;41(11):1275-93.

[32] Rodriguez A, Bohlin G. Are maternal smoking and stress during pregnancy related to ADHD symptoms in children? J Child Psychol Psychiatry 2005;46(3):246-54.

[33] Van den Bergh BRH, Marcoen A. High antenatal maternal anxiety is related to ADHD symptoms, externalizing problems, and anxiety in 8- and 9-year-olds. Child Dev 2004;75(4):1085-97.

[34] O'Connor TG, Heron J, Glover V. Antenatal anxiety predicts child behavioral/emotional problems independently of postnatal depression. J Am Acad Child Adolesc Psychiatry

2002;41(12):1470-7.

[35] O'Connor TG, Heron J, Golding J, Beveridge M, Glover V. Maternal antenatal anxiety and children's behavioural/emotional problems at 4 years. Br J Psychiatry 2002;180:502-8.

[36] Hirshfeld-Becker DR, Biederman J, Faraone S, Robin JA, Friedman D, Rosenthal JM, et al. Pregnancy complications associated with childhood anxiety disorders. Depress Anxiety 2004;19:15262.

[37] Beesdo K, Pine DS, Lieb R, Wittchen H-U. Incidence and risk patterns of anxiety and depressive disorders and categorization of general anxiety disorder. Arch Gen Psychiatry 2010;67(1):1-12.

[38] Bittner A, Egger HL, Erkanli A, Costello EJ, Foley DL, Angold A. What do childhood anxiety disorders predict? J Child Psychol Psychiatry 2007;48(12):1174-83.

[39] Brückl TM, Wittchen H-U, Höfler M, Pfister H, Schneider S, Lieb R. Childhood separation anxiety and the risk of subsequent psychopathology: results from a community study. Psychother Psychosom 2007;76(1):47-56.

[40] Last CG, Perrin S, Hersen M, Kazdin AE. A prospective study of childhood anxiety disorders. J Am Acad Child Adolesc Psychiatry 1996;35(11):1502-10.

[41] Realmuto GM, Winters KC, August GJ, Lee S, Fahnhorst T, Botzet A. Drug use and psychosocial functioning of a community-derived sample of adolescents with childhood ADHD. J Child Adolesc Subst Abuse 2009;18(2):172-92.

[42] Lieb R, Isensee B, von Sydow K, Wittchen H-U. The Early Developmental Stages of Psychopathology Study (EDSP): a methodological update. Eur Addict Res 2000;6(4):170-82. [43] Wittchen H-U, Perkonigg A, Lachner G, Nelson CB. Early Developmental Stages of Psychopathology Study (EDSP)—objectives and design. Eur Addict Res 1998;4(1-2):18-27. [44] Lachner G, Wittchen H-U, Perkonigg A, Holly A, Schuster P, Wunderlich U, et al. Structure, content and reliability of the Munich Composite International Diagnostic Interview (M-CIDI) substance use sections. Eur Addict Res 1998;4(1-2):28-41.

[45] Reed V, Gander F, Pfister H, Steiger A, Sonntag H, Trenkwalder C, et al. To what degree does the Composite International Diagnostic Interview (CIDI) correctly identify DSM-IV disorders? Testing validity issues in a clinical sample. Int J Methods Psychiatr Res 1998;7(3):142-55. [46] Wittchen H-U, Pfister H. DIA-X-Interviews: Instruktionsmaterial zur Durchführung von DIA-X Interviews. Frankfurt: Swets \& Zeitlinger; 1997.

[47] Berkowitz GS, Papiernik E. Epidemiology of preterm birth. Epidemiol Rev 1993;15(2):414-43. [48] Apgar V. A proposal for a new method of evaluation of the newborn infant. Curr Res Anesth Analg 1953;32(4):260-7.

[49] Stata. Stata Statistical Software. 2007.

[50] Royall M. Model robust confidence intervals using maximum likelihood estimations. Int Stat Rev 1986;54(2):221-6.

[51] Savitz DA, Olshan AF. Multiple comparisons and related issues in the interpretation of epidemiologic data. Am J Epidemiol 1995;142(9):904-8.

[52] Murray L, Cooper P, Creswell C, Schofield E, Sack C. The effects of maternal social phobia on mother-infant interactions and infant social responsiveness. J Child Psychol Psychiatry

2007;48(1):45-52. 
[53] Walshe M, Rifkin L, Rooney M, Healy E, Nosarti C, Wyatt J, et al. Psychiatric disorder in young adults born very preterm: role of family history. Eur Psychiatry 2008;23(7):527-31.

[54] Hodgson DM, Coe CL. Perinatal programming: early life determinants of adult health and disease. London: Taylor \& Francis; 2005.

[55] Mulder EJH, de Medina PGR, Huizink AC, Van den Bergh BRH, Buitelaar JK, Visser GHA.

Prenatal maternal stress: effects on pregnancy and the (unborn) child. Early Hum Dev 2002;70:3-14. [56] Teixeira JMA, Fisk NM, Glover V. Association between maternal anxiety in pregnancy and increased uterine artery resistance index: cohort based study. Br Med J 1999;318:153-7.

[57] Bhutta AT, Cleves MA, Casey PH, Cradock MM, Anand KJS. Cognitive and behavioural outcomes of school-aged children who were born preterm: a meta-analysis. JAMA 2002;288(6):72837. 